\title{
Management of Oral Manifestations in a Case of Cushing's Habitus
}

\author{
${ }^{1}$ Mohinder Panwar, ${ }^{2}$ Jacqueline J Dias, ${ }^{3}$ Rohit Sharma
}

\section{ABSTRACT}

Background: Cushing's syndrome is a debilitating endocrine disorder comprising signs and symptoms due to prolonged exposure to cortisol. It is characterized by excessive cortisol levels in the blood, which may be the result of various causes, such as tumors of pituitary glands, tumors arising elsewhere in the body (ectopic adrenocorticotropic hormone-producing tumors), or exogenous causes, such as prolonged intake of steroids (known as Cushing's habitus).

Aim: This article is a case report on the management of a patient with Cushing's habitus, presenting with recurrent oral ulcers as one of the manifestations.

Case report: A 25-year-old patient diagnosed with Cushing's habitus came to the department with complaint of recurrent ulcers on the tongue and lips since 6 months. These ulcers were unresponsive toward conventional therapies, such as amlexanox and steroids.

Conclusion: Due to the unique history presented by the patient and the persistence of recalcitrant ulcers, an immunomodulator was prescribed. This drug prevented the recurrence of the oral ulcers.

Clinical significance: Various manifestations of Cushing's disease include metabolic, cardiovascular, and endocrinal abnormalities, to mention a few. Prevalence of dental manifestations is rare, oral ulcers being among the few. These oral ulcers require special consideration since the underlying pathogenesis of the ulcers is very different. This article describes the management of such ulcers in Cushing's habitus using an immunomodulator.

Keywords: Cushing's habitus, Immunomodulator, Recurrent oral ulcers, Thalidomide.

How to cite this article: Panwar M, Dias JJ, Sharma R. Management of Oral Manifestations in a Case of Cushing's Habitus. Int J Experiment Dent Sci 2018;7(1):35-38.

Source of support: Nil

Conflict of interest: None

${ }^{1}$ Professor, ${ }^{2}$ Postgraduate Resident, ${ }^{3}$ Assistant Professor

1,2Department of Periodontology and Department of Dental Surgery and Oral Health Sciences, Armed Forces Medical College, Pune Maharashtra, India

${ }^{3}$ Department of Oral Surgery, Armed Forces Medical College Pune, Maharashtra, India

Corresponding Author: Jacqueline J Dias, Postgraduate Resident, Department of Periodontology and Department of Dental Surgery and Oral Health Sciences, Armed Forces Medical College, Pune, Maharashtra, India, Phone: +919971579012 e-mail: jacintadias.90@gmail.com

\section{BACKGROUND}

An ulcer can be defined as a breach in the integrity of epithelium, surface, or mucosa. Oral ulcers are one of the most commonly occurring lesions in the oral cavity. Prevalence of oral ulcers is around $4 \%$ worldwide, ${ }^{1}$ the most common being recurrent aphthous ulcers, having a prevalence of around $21.7 \%$ in India. Some of the systemic diseases presenting oral ulcers as clinical manifestations include infections, immunological diseases, and trauma, to mention a few. There are also certain conditions that infrequently manifest as oral ulcers, one of them being Cushing's syndrome. Cushing's syndrome is a hormonal disorder comprising a group of signs and symptoms due to chronic exposure to abnormally elevated levels of plasma glucocorticoid. There are numerous causes for Cushing's syndrome, the commonest being exogenous steroid (Cushing's habitus) and pituitary adenoma (Cushing's disease). ${ }^{2}$

\section{CASE REPORT}

A 25-year-old male patient came to the department with complaints of recurring ulcers on tongue and lips since 6 months.

Patient gave a history of injury during contact sports (basketball) 4 years back, resulting in laceration of the upper lip. Patient visited a private hospital for the laceration and was prescribed betamethasone $10 \mathrm{mg}$, three times a day for 1 month. However, the patient continued the medication for 4 years without any medical consultation.

One year back, the patient gained weight and subsequently developed purple striae on abdomen and chest (Fig. 1).

Patient then reported to the Department of Endocrinology where laboratory investigations were performed and patient's cortisol level was $191.33 \mu \mathrm{mol} / \mathrm{L}$. The patient was diagnosed with Cushing's habitus secondary to exogenous steroid. He was prescribed hydrocortisone, $20 \mathrm{mg}$, twice a day for 10 days. After that, the drug was tapered to hydrocortisone, $10 \mathrm{mg}$ twice a day for 3 days and then further tapered to $10 \mathrm{mg}$, once a day for 3 days. After this, the drug was changed from hydrocortisone to prednisolone, $5 \mathrm{mg}$, twice a day along with calcium sachets. Patient was then referred to the Department of Dental Surgery for management of oral ulcers. 


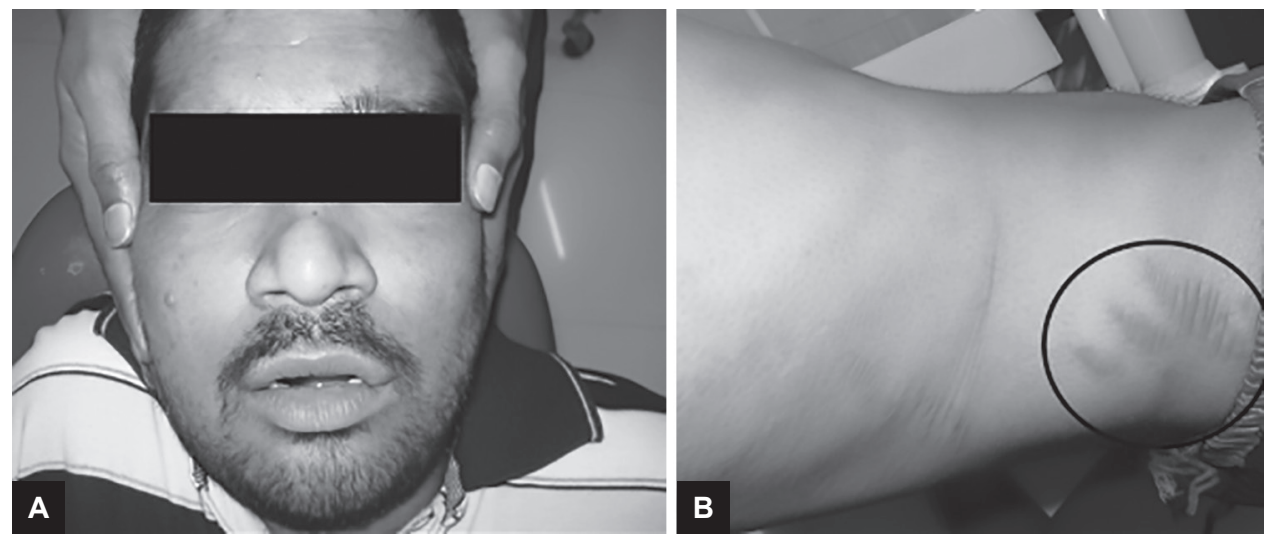

Figs 1A and B: Purple striae on abdomen, chest, and moon facies
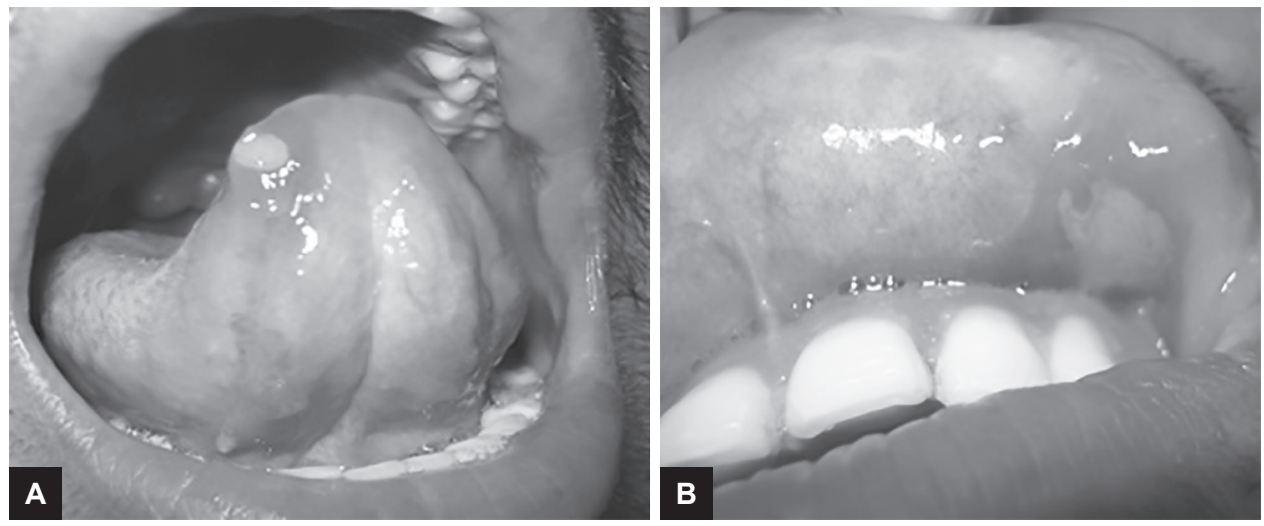

Figs 2A and B: Ulcer on ventral surface of tongue, upper labial mucosa
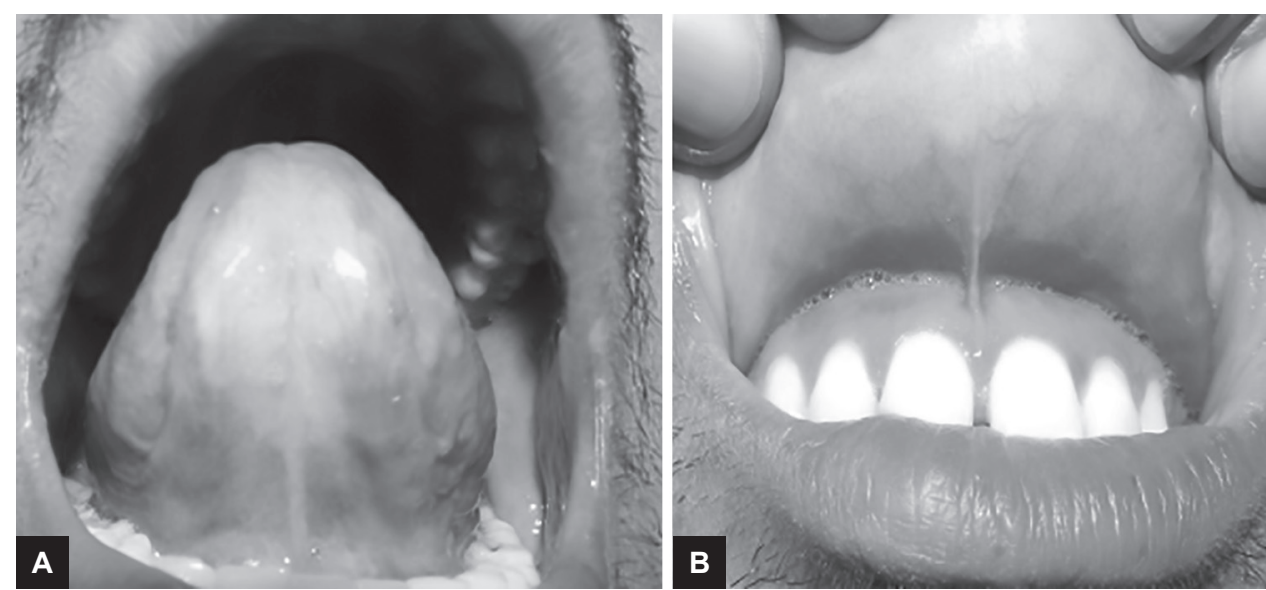

Figs $3 A$ and B: Two weeks posttreatment

On intraoral examination, multiple ulcers measuring $1.5 \mathrm{~cm}$ with round, yellow depressed area surrounded by erythema on the ventral surface of tongue and upper and lower labial mucosa were noted. The ulcers had sloping edges, irregular margins, and were tender on palpation (Fig. 2).

Based on the history presented and clinical findings, a provisional diagnosis of oral ulcers due to Cushing's habitus secondary to exogenous steroid was given.
Patient was prescribed dologel for symptomatic relief along with $5 \%$ amlexanox. There was no relief of symptoms.

The patient was then prescribed cap thalidomide $50 \mathrm{mg}$ twice a day for 2 weeks after consultation with the endocrinologist. Review was done after 2 weeks and there was reduction in size and numbers of ulcers (Fig. 3). The drug was then tapered to cap thalidomide, $50 \mathrm{mg}$ once a day for 2 more weeks, and then stopped after investigating 

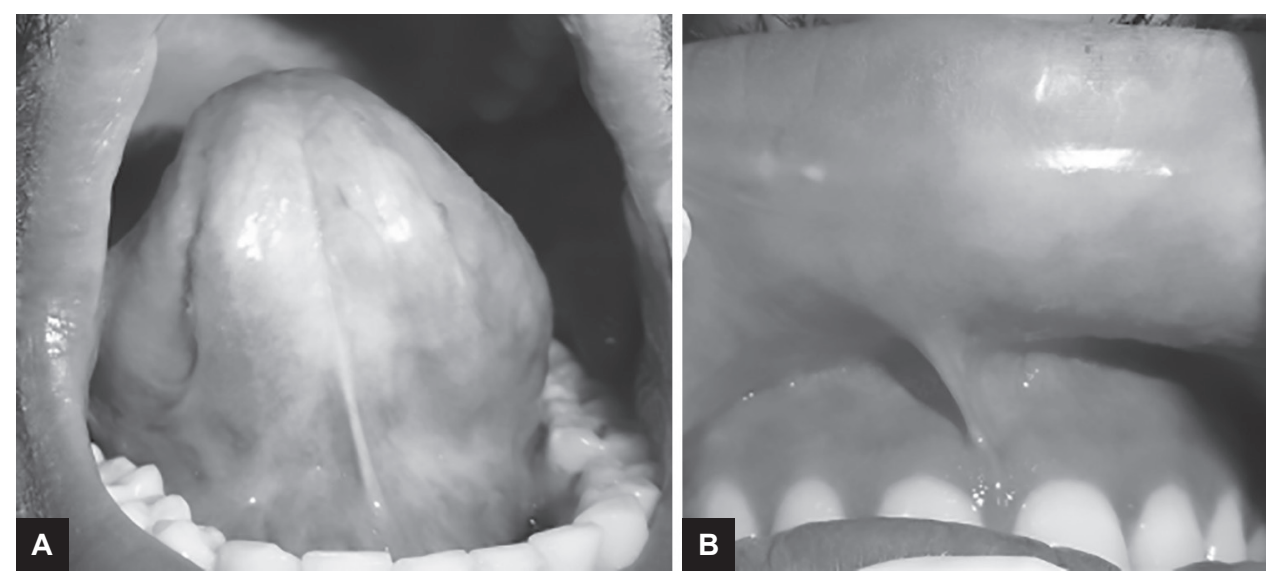

Figs 4A and B: Two months posttreatment

the cortisol level again. The ulcers had healed completely. After 2 months, review was done and there was no recurrence of ulcers in the oral cavity (Fig. 4).

\section{DISCUSSION}

Oral ulcers can be due to numerous reasons. They can be the manifestation of various systemic diseases, such as infections, immunological disorders, trauma, and allergic reactions, to mention a few. ${ }^{3}$ Understanding the pathophysiology of ulcers becomes indispensable for correct management. Often, the history and clinical presentation of ulcers, such as margins, slopes, and edges direct at giving a better provisional diagnosis.

It is interesting to note that quite often, immune response of an individual plays a vital role in the pathogenesis of an ulcer. Even in ulcers, such as those caused due to infection, trauma, and allergy, the immune cells play a substantial role in the destruction of the epithelium. The immune cells, such as neutrophils and lymphocytes release various cytokines in response to stimulus. These cytokines, such as interleukin (IL-1), IL-6, and tumor necrotic factor alpha ${ }^{4}$ are responsible for destruction of cell-cell interaction via intercellular adhesion molecule 1 and other cell adhesion molecules, resulting in ulcerations. ${ }^{5}$

The commonest modality for management of an oral ulcer comprises elimination of obvious etiology apart from providing the patient with symptomatic relief, such as local anesthetics and anti-inflammatory therapy like chlorhexidine and tetracycline mouthwash to reduce the microbial load. ${ }^{6}$ One of the frequently employed management involves prescription of steroid for ulcers. Steroids are immunomodulators that suppress the immune response. Expanding knowledge on the imperative role of immune response in the pathophysiology of ulcer has resulted in growing popularity of immunomodulator drugs as a treatment modality. ${ }^{7}$

Immunomodulators can be immunostimulants or immunosuppressants depending on their mechanism of action. Immunosuppressants like steroids, azathioprine, and tacrolimus can be prescribed in oral ulcers due to exaggerated immune response, such as in pemphigus and lichen planus. Immunostimulants can be prescribed in oral ulcers due to suppression of immune cells. ${ }^{8}$

Cushing's syndrome comprises vast clinical manifestations ranging from metabolic abnormalities, endocrine dysfunction, cardiovascular abnormalities, dermatological changes, infections, obesity, thin mucosa, and many more. Even then, the prevalence of dental manifestations documented in literature is very rare. Recurrent ulcers as clinical manifestation of Cushing's syndrome have been seldom reported. ${ }^{9}$

Immunomodulators that stimulate the immune response, such as levamisole, thalidomide, and bacillus Calmette-Guerin can be prescribed in such cases. These immunostimulants modulate and escalate the immune system. Therefore, immunomodulators, specifically immunostimulants, can be prescribed for management of oral ulcers in Cushing's syndrome. ${ }^{10}$

However, immunomodulators are associated with adverse effects, such as risk of infection, myelosuppression, hepatotoxicity; hence, the risk-benefit ratio has to be taken into consideration. Prolonged use of immunomodulators is avoided due to these side effects. Regular blood and liver function investigations are essential. Also, understanding the mechanism of individual drug becomes vital for appropriate usage and prescription.

\section{CONCLUSION}

Oral ulcers are one of the rare oral manifestations of Cushing's syndrome. The management of oral ulcers in Cushing's would involve understanding the underlying pathophysiology. Immunomodulators have a bright future in the management of oral ulcers caused due to altered immune response; however, understanding the mechanism becomes significant for correct usage of these drugs. Due to low prevalence of oral ulcers 
expressing as manifestation in Cushing's, very few literature substantiates the use of immunomodulators. Hence, studies to comprehend the long-term benefits and side effects of use of immunomodulators in oral ulcers become imperative.

\section{CLINICAL SIGNIFICANCE}

Our patient formerly diagnosed with Cushing's habitus presented with recurrent ulcers. Managing a patient with Cushing's syndrome involves managing the various comorbidities associated with it, such as hypertension, diabetes mellitus, and cardiovascular abnormalities, to mention a few. Oral ulcers are one of the rare oral manifestations in Cushing's disease. These ulcers have to be managed keeping in mind its pathophysiology (atrophy of epithelium associated with immune suppression in Cushing's disease). This article highlights the significant role immunomodulators can play in such unique scenarios.

\section{REFERENCES}

1. Shulman JD, Beach MM, Rivera-Hidalgo F. The prevalence of oral mucosal lesions in U.S. adults: data from the Third National Health and Nutrition Examination Survey, 19881994. J Am Dent Assoc 2004 Sep;135(9):1279-1286.
2. Nieman LK, Biller BM, Findling JW, Murad MH, Newell-Price J, Savage MO, Tabarin A. Treatment of Cushing's syndrome: an endocrine society clinical practice guideline. J Clin Endocrinol Metab 2015 Aug;100(8):2807-2831.

3. Long RG, Hlousek L, Doyle JL. Oral manifestations of systemic diseases. Mt Sinai J Med 1998 Oct-Nov;65(5-6):309-315.

4. Buno IJ, Huff JC, Weston WL, Cook DT, Brice SL. Elevated levels of interferon gamma, tumor necrosis factor alpha, interleukins 2, 4, and 5, but not interleukin 10, are present in recurrent aphthous stomatitis. Arch Dermatol 1998 Jul;134(7):827-831.

5. Kose O, Stewart J, Waseem A, Lalli A, Fortune F. Expression of cytokeratins, adhesion and activation molecules in oral ulcers of Behcet's disease. Clin Exp Dermatol 2008 Jan;33(1):62-69.

6. Altenburg A, Abdel-Naser MB, Seeber H, Abdallah M, Zouboulis CC. Practical aspects of management of recurrent aphthous stomatitis. J Eur Acad Dermatol Venereol 2007 Sep;21(8):1019-1026.

7. Shivhare P, Shankarnarayan L, Singh A, Patil ST, Yadav M. Role of immunomodulators in oral diseases. Int J Oral Health Med Res 2015;2(3):73-80.

8. Elad S, Epstein JB, Yarom N, Drucker S, Tzach R, von Bultzingslowen I. Topical immunomodulators for management of oral mucosal conditions, a systematic review. Part I: Calcineurin inhibitors. Expert Opin Emerg Drugs 2010 Dec;15(4):713-726.

9. Carlos Fabue L, Soriano YJ, Sarrion Perez MG. Dental management of patients with endocrine disorders. J Clin Exp Dent 2010;2(4):e196-e203.

10. Bascones-Martinez A, Mattila R, Gomez-Font R, Meurman JH. Immunomodulatory drugs: oral and systemic adverse effects. Med Oral Patol Oral Cir Bucal 2014 Jan;19(1):e24-e31. 